\author{
Alternating Gradient Synchrotron Department \\ Brookhaven National Laboratory \\ Associated Universities, Inc. \\ Upton, New York 11973
}

Experimental Planning and Support Division Technical Note

AGS/EP\&S/Technical Note 131

November 10, 1988

\title{
HIGH INTENSITY TARGET STATION STUDY: PHASE I
}

D. Beavis. A.S. Carroll, W. Leonhardt, J. Mills, A. Pendzick, E. Schwaner 


\title{
HIGH INTENSITY TARGET STATION STUDY \\ PHASE I
}

\author{
D. Beavis, A. S. Carroll, W. Leonhardt \\ J. Mills, A. Pendzick, E. Schwaner
}

\section{INTRODUCTION :}

This Phase I report is the first of three proposed reports on the requirements for high intensity target stations, those capable of accepting beams from the AGS after the completion of the Booster. The high intensity target stations in this new era will have incident beam intensities in the range of 1 to $5 \times 10 * * 13$ protons/pulse. We will attempt to define the expected radiation patterns around an extracted beam production target. From these patterns, the areas requiring radiation hardening beyond present practices can be determined. Then the zones of applicability of commonly used materials are determined from their radiation resistance. For the benefit of future target station designers, an anecdotal list of common failures is given. Possible inorganic insulations are considered as to their cost and availability. Finally these ideas are applied to the present $\mathrm{C}$ target station to assess their impact. These issues are of considerable importance because reliability leads to more efficient running of the AGS and in reduced radiation exposure to department personnel. A summary of instrumentation needs is also included.

Target stations under design or construction at this time such as the C target upgrade, the g-2 target station, and the $2 \mathrm{GeV} / \mathrm{c}$ beam target station will still be in operation after the Booster is completed. Their design will be considered in the Phase II report.

The Phase III report will present a SEB "Master Plan" which will suggest an scenario for as orderly a development as possible for the AGS target stations, given the large uncertainties in predicting the future experimental program.

\section{TARGET STATION RADIATION PATTERNS:}

A conservative approximation to the radiation pattern around an AGS production target can be obtained by considering the radiation from a bare, unshielded target. Shielding will generally reduce the flux (particles/cm**2) reaching a given point, and hence the radiation dose. This is true for all but the most forward angles(see Fig. 4).

The radiation pattern from a typical AGS platinum production target, 7.5 $\mathrm{cm}$ long and $5 \mathrm{~mm}$ diameter, was calculated by A. Stevens using the CASIM nuclear cascade simulation program\{1\}. The contours of equal radiation dose are shown in Fig. 1 for an incident intensity of $10 \star \star 13$ protons/pulse for a 
period of 15 weeks. This represents about twice the present integrated incident beam for $a$ year at $C$ target station. With the Booster, the integrated flux would be $u$ p by another factor of 2.5. The radiation resistance of good organic insulators is about $10 * * 9$ rads, so that the expected lifetime in the inner zone is less than one year.\{2\} Since the dose falls off rapidly with distance from the beam center line, good organic insulators are generally satisfactory for all but the more forward and close in components. The radiation upstream of the target $(>12$ inches, $30 \mathrm{~cm}) \mathrm{falls}$ off extremely rapidly, and thus requires no special consideration unless the beam is likely to scrape on components further upstream of the target. J. W. Glenn reports that radiation damage to components upstream of the target has not been a problem thus far.

The expected radiation dose in a dipole placed just downstream of a target has been calculated by D. Beavis using the CASIM simulation program. As shown in Fig. 2, the radiation dose to coils is sufficient to cause failure in organically insulated coils in less than one year at post Booster intensities.

The radiation pattern was also calculated with CASIM with two quadrupoles and a thick shielding wall in the forward direction(see Fig 3). The magnetic fields of the quadrupoles have little effect (less than a factor of 2) and can be neglected in calculating the longevity of components in a radiation field.

As a guide to the effectiveness of shielding, Fig. 4 displays the results of CASIM calculations of the attenuation radiation dosage in steel for particles leaving the production target at various angles from 1 to 20 Degrees. The depth is measured along the trajectory of the particle. For angles greater than 20 Degrees a reasonable rule of thumb is a reduction by a factor of 2 every 5 inches $(12.5 \mathrm{~cm})$. The approximate effectiveness of other materials may estimated by scaling by their density. These curves should be used with caution at angles less than 5 Degrees and distances less than a few feet. If the application is critical or the component is near a shielding edge, then a CASIM calculation should be done. Note that certain types of shielding such as lead,tungsten, and uranium should not be exposed to the direct proton beam because they may melt( $\mathrm{Pb})$, disintegrate(W) or overheat(U).

These radiation patterns have been checked by placing aluminum and copper discs around the "C" production target. The observed doses and comparisons with calculations will be the subject of a future report.

\section{RADIATION RESISTANCE:}

Table I contains a list of commonly used materials at AGS target stations. There is a large range of radiation resistance, so care should be exercised in choosing the best material. Materials not on the list, such as Teflon, generally have even lower radiation resistance $(<10 * * 6$ Rads) and should be avoided. It is generally believed that materials will not fail before they have received at least "moderate" radiation damage. If the radiation resistance of a new material is unknown, BNL can test the material 
using BLIP or the HFBR.

\section{IMPROVED RADIATION RESISTANT INSULATORS:}

The most vulnerable materials around production targets are the organic insulators on magnet windings, magnet power cables, control and interlock wiring, and water hoses. If we could afford to replace all of these organic insulators with inorganic ones, there would be no problems with radiation damage except for materials directly exposed to the primary proton beam. A typical inorganic insulator has a radiation resistance of about $10 * * 12$ Rads. However, such materials are generally much more expensive to manufacture, more difficult to install, and more prone to mechanical failure.

By choosing the best of the organic insulators in Table I, for example Kapton or epoxy-fiberglass, it is possible to use these materials in all but the immediate areas downstream of the target. An adequate solution can frequently be found by using a combination of methods. For example, metal piping can be employed in the highest radiation area, and then normal hoses can be connected for the remainder of the run.

There are flexible ceramic insulated wires suitable for instrumentation and control wiring $\{3\}$. These would be used sparingly, since they are expensive ( $\$ 10 / f o o t)$ and only available in aluminum. For somewhat lower level radiation ( $<10 * * 10$ rads), Kyle Technology makes Kapton insulated wires. $\{4\}$

Most magnets are connected with 500 MCM locomotive cable. The old cable used an undocumented insulation, probably a rubber/neoprene. More recently we have switched to HYPALON insulation which has rating of over $2 \times 10 * * 7$ Rads. Very recent investiagations have located a cross-linked(by radiation!) polyolefin which has a rating over $2 \times 10 * * 8$ Rads. The later is a cable used in the nuclear power industry, a good source of radiation resistant materials. Even higher resistance can be achieved with KAPTON, but the mechanical strength is uncertain.

Septum magnets and quadrupoles in areas just downstream of production targets need to be replaced every two to four years at present AGS intensities. With a factor of five increase, their mean life will drop below one year's usage. Also the area radiation levels will be five times higher than at present. Clearly, the present epoxy- fiberglass insulated coils need to be replaced with inorganic insulation in these locations. TRIUMF and LAMPF both have successfully used a jacketed copper conductor with an insulating mineral powder (magnesium oxide) inside the jacket $\{5\}$. This insulation is relatively inexpensive, flexible and easy to wind. Its main drawback is that the maximum current density is $45 \%$ of our present current density. $W$. Leonhardt is investigating whether an anodized aluminum cladding on a copper conductor might provide a satisfactory solution. Insulators made from ceramic coatings have been used, but these are expensive and difficult to manufacture. As with any new technology, considerable development will be required, and there is a good likelihood of some initial failures. We should try to settle on one or at most two technologies for this application. 
The use of conventional insulations may be possible if the coils are kept well away from the magnet midplane. It is possible that if the moisture could be kept away from the magnet coils, their effective lifetime would be considerably extended.

COMMON FAILURE MODES:

In Table II, many of the common failures at target stations are listed. The list is approximately ordered in terms of perceived importance. No claim is made for completeness or scientific sampling. This list is intended to indicate the types of failure which might occur and to suggest possible corrective steps. Hopefully, it will at least prevent the reoccurence of old mistakes.

Many of the failures are not radiation damage failures, but become radiation exposure problems when the repairs need to be made. This points to the need to be extra cautious in terms of cooling water quality, interlock and circuit breaker design, and other ancillary equipment design in these areas. Means for remotely diagnosing possible faults is also an important consideration. An example of remote diagnosis technique is the installation of voltage taps on magnet coils to detect both insulation faults and overheating. Remote viewing of the target stations with video or infr ared cameras might be another possibility.

The requirements and problems associated with instrumentation at high intensity target stations are summarized in Table III. More details are to be reported in future Tech Notes.

COST GUIDELINES FOR RADIATION HARDENING:

If the reliabity of components in radiation areas can be increased or their replacement made quicker, then the radiation exposure of AGS staff is reduced. However, at some point, the cost of more reliability or ease in replacement will be excessive. Spending $\$ 100 \mathrm{~K}$ to reduce a $100 \mathrm{mRem}$ exposure to $75 \mathrm{mRem}$ is definitely excessive. A 1980 report put the reasonable figure for reducing radiation exposure by one Rem to be in the range of $\$ 3 \mathrm{~K}$ to $\$ 80 \mathrm{~K}$. $\{6\}$ At the lower limit, the changes should automatically be done and at the upper limit, changes generally wouldn't be implemented. Something in the midrange of approximately $\$ 20 \mathrm{~K}$ seems reasonable. Since a majority of failed components can not be repaired after irradiation, each occurence results in an equipment replacement cost which is often tens of thousands of dollars. "Serious" failures at the "C" target station have typically resulted in exposures of 0.5 to 5 Rem and one to two weeks of AGS downtime.

All this says that it is worth a lot of thought and a considerable expense to reduce radiation exposure. Such efforts over the past decade have reduced the department exposure by a factor of 6 despite the increases in AGS intensity. Using the techniques suggested in this and future reports, we need to make another factor of ten reduction by the time that the Booster is operational. 


\section{ACKNOWLEDGEMENTS :}

We wish to thank A. Stevens for carrying out some of the CASIM calculations, and I-H Chiang for supplying the data on Instrumentation in table III. D. Lazarus supplied many helpful suggestions.

\section{REFERENCES}

1. A. J. Stevens "Improvements in CASIM; Comparison with Data", AGS/AD/Tech Note No. 296(1988); A. Van Ginneken, "CASIM Program to Simulate Hadronic Cascades in Bulk Matter", Fermilab FN-272 (1975)

2. M. H. Van de Voorde and C. Restat, "Selection Guide to Organic Materials for Nuclear Engineering"

3. Data sheet, Kurt J. Lesker Co., "Pliable Ceramic-coated Wire for UHV Applications"(1988)

4. Kyle Technology, Roseburg, Oregon

5. Pyrotenax of Canada Ltd, Trenton, Ontario, Canada

6. "A Guide to Reducing Radiation Exposure to As Low As Reasonably Achievable(ALARA)", DOE/EV/1830-T5 (1980) 
Table I. Radiation Resistance of Commonly Used Materials at AGS Target Stations*

TRADE NAME

CHEMICAL NAME

MILD

MODERATE

DAMAGE DAMAGE

(RADS) (RADS)

\begin{tabular}{|c|c|c|c|}
\hline micarta & phenolic & $2 \times 10 * * 6$ & $2 \times 10 * * 7$ \\
\hline polypenco & polystyrene(solid) & $7 \times 10 * * 8$ & $9 \times 10 * * 9$ \\
\hline plexiglass & acrylic resin & $2 \times 10 * * 5$ & $8 \times 10 * * 5$ \\
\hline epoxy & epoxy resin & $3 \times 10 * * 8$ & $8 \times 10 * * 8$ \\
\hline \multicolumn{4}{|l|}{ klixon- } \\
\hline phenolic base & phenolic & $2 \times 10 * * 6$ & $2 \times 10 * * 7$ \\
\hline rubber insul. & nat. polyisoprene & $3 \times 10 * * 7$ & $1 \times 10 * * 8$ \\
\hline polyethylene & polye thylene & $1 \times 10 * * 7$ & $9 \times 10 * * 7$ \\
\hline kapton & polyimide & $1 \times 10 * * 8$ & $1 \times 10 * * 10$ \\
\hline \multicolumn{4}{|l|}{ o-rings } \\
\hline neoprene & neoprene & $2 \times 10 * * 7$ & $1 \times 10 * * 8$ \\
\hline butyl & butyl & $9 \times 10 * * 6$ & $6 \times 10 * * 6$ \\
\hline \multicolumn{4}{|c|}{ synflex water hose } \\
\hline nylon core & polyamide & $6 \times 10 * * 5$ & $2 \times 10 * * 6$ \\
\hline polyester & & $3 \times 10 * * 5$ & $3 \times 10 * * 6$ \\
\hline insulation & polyethylene & $1 \times 10 * * 7$ & $9 \times 10 * * 7$ \\
\hline \multicolumn{4}{|l|}{ W/c buss- } \\
\hline PVC insulator & polyvinylchloride & $9.5 \times 10 * * 6$ & $9 \times 10 * * 7$ \\
\hline \multicolumn{2}{|l|}{ coaxial cable- } & $9.5 \times 10 * * 6$ & $9 \times 10 * * 7$ \\
\hline insulation & polye thylene & $1 \times 10 * * 7$ & $9 \times 10 * * 7$ \\
\hline $\begin{array}{l}\text { nylon cable-ties } \\
\text { G-10 insulator }\end{array}$ & \multicolumn{2}{|c|}{ G-10 insulator } & $2 \times 10 * * 6$ \\
\hline \multicolumn{4}{|l|}{500 MCM locomotive } \\
\hline cable & hypalon & $2 \times 10 * * 7$ & $9 \times 10 * * 7$ \\
\hline exane & $\begin{array}{l}\text { cross-linked } \\
\text { polyolefin }\end{array}$ & $2 \times 10 * * 8$ & $1 \times 10 * * 9($ unofficial) \\
\hline
\end{tabular}

*The major source used for this table was a report from CERN ISR Division, SELECTION GUIDE TO ORGANIC MATERIALS FOR NUCLEAR ENGINEERING. M.H.Van de Voorde and C. Restat. As stated in the report, most of the data was compiled using gamma source and nuclear reactor irradiation.

The authors view the term mild damage to mean that the material is slightly radiation damaged but often useable. The term moderate damage is assumed to mean the material is questionable and should be considered to be of limited use. 
Table II. Common Failures at Target Stations

FAILURES(in order of decreasing freq.) | POSSIBLE SOLUTIONS

1. Water Hose Damage

2. Interlock cable damage

3. Magnet coils

4. 500 MCM magnet power cables

5. Vacuum seals

6. Water seals(organic materials)

7. Butterball valve leakage

8. Klixon wiring

9. D. C. Cover boxes

10. H-film damage

11. Damage to Micarta Bus Blocks

12. Thermocouple wire

13. Lighting

14. Targets and thermocouples

15. Fire alarm cable damage
Investigate new ceramics

Move away from high radiation zones

Use ceramic cables and connectors Move away from high radiation zones

Investigate anodized aluminum and magnesium oxide insulators

Move coils away from magnet midplane

Use cross linked polyolefin insulator: rad. re Use non insulated cables

Consider new welded systems Investigate new Marmon clamps

Try plumbing unions at magnet

Investigate hard seat valves

Use ceramic coated wire

Use higher radiation resistent wire

Use aluminum boxes

Use no boxes

Try anodized aluminum??

New Materials - polypenco

Use pigtail to target

Use ceramic bases on incandescent bulbs upstream

Use radiation hard cables

Use new air cooled design Secure thermocouple by peaning

Split feed at target. Investigate long range smoke detectors (light beam detectors) 
Table III. Instrumentation for High Intensity Target Stations

1. Intensity monitors: We use SEC(Secondary Emission Chambers) to monitor the beam intensity when beam intensity exceeds $10 * \star 11$. For this device to function properly, it has to be operated in a very high vacuum environment. The present design requires that it is pumped by the Ion pump continuously. The SEC needs to be calibrated occasionally. The $\mathrm{C}$ target SEC was in operation for at least 3 SEB runs and the calibrations are reasonable. We did not have enough data to establish exactly how stable it is, but the indications are that they are within $20 \%$ of the initial condition. This device was designed to couple to the vacuum pipe with its own window. For now we can not plunge them in the external vacuum chambers. A quick replacement mechanism should be provided. Also a mechanism should be devised to install and retrieve the foil for calibration.

2. Interaction monitor: For the SEB, we use the $90 \mathrm{deg}$ telescope to monitor the interaction rate. For high intensity, we have to vary the size of the counters to limit the rate of the counters. For the FEB, a $90 \mathrm{deg}$ ion counter can be used for this purpose.

3. Beam position servo: There are two types of devices used for this purpose. SWIC and Split plates ion chamber. The SWIC is more sensitive and used in almost all the servo magnets now. For high intensity, the SWIC's are more susceptible to radiation damage. The Split plate ion chamber can be improved for use in the high intensity station, for example by enclosing them in the controlled gas environment. In principle, a split plate is more rugged and more immune to radiation damage.

4. Position monitor: Normally, we use aluminum oxide flags and SWICs for this purpose. The flag used at the $\mathrm{C}$ target station, only lasted for about 2 weeks at full intensity.There is a conjecture that if they were in vacuum or in helium, they might last longer. There are no data to support this yet. The SWICs are a little better, but if the beam is targetted on the G-10 flange too long, the leakage current will make the SWIC useless. After the first month of the last SEB operation, both SWIC's and Flags were in bad shape. To eliminate some of the problems, we should install dual plunging SWIC's upstream of target stations to define the incident angle. In addition, we should improve the SWICs to eliminate the tails present for high intensity running. One possibilty is to use the residual gas ionization SWIC. This has been tested in the $\mathrm{C}$ line. Some gold plated tungsten wires were broken after 2 months of beam time. This can be remedied by using nickel wire and plunging the SWICs. For these devices to be effective, we need to have a better control system to calculate the beam size and position at the target. For the direct observation of the beam size at the target, we need to provide a better SWIC and to develop some kind of quick replacement mechanisms. 


\section{CONTOURS OF RADIATION DOSE NEAR A SEB TARGET $\left(10^{13}\right.$ Protons/pulse for 15 weeks)}

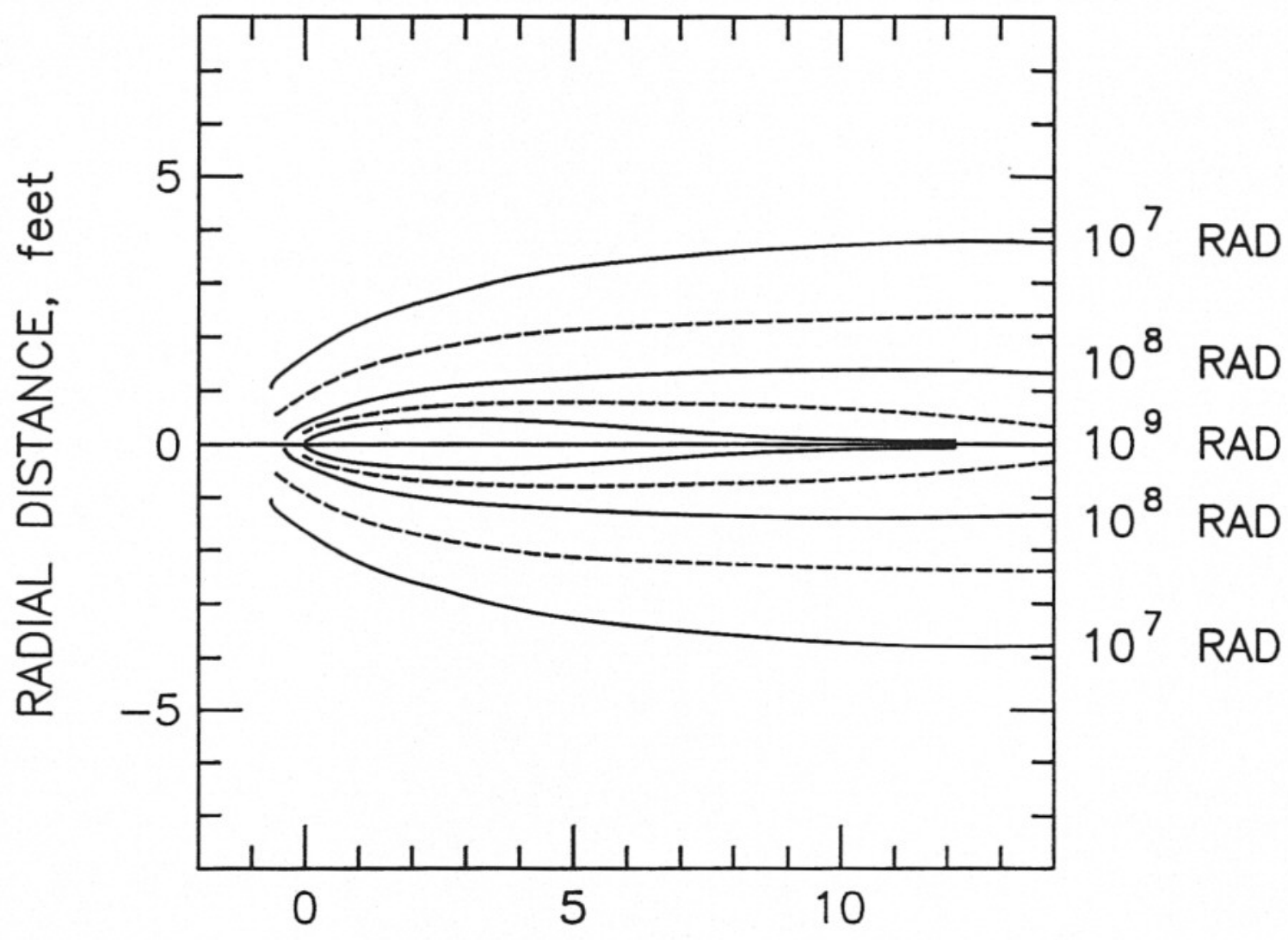

LONGITUDINAL DISTANCE, feet 
RADIUS (inches)



Fig. 2. Expected radiation dose for a dipole placed just downstream of a production target. Incident flux was $10^{13}$ protons/pulse for 15 weeks. 




Fig. 3. The radiation pattern with two quadrupoles, with and without iron shield walls. 
NORMALIZED RROTRTION DOSE VS DEFTH IN STEEL,

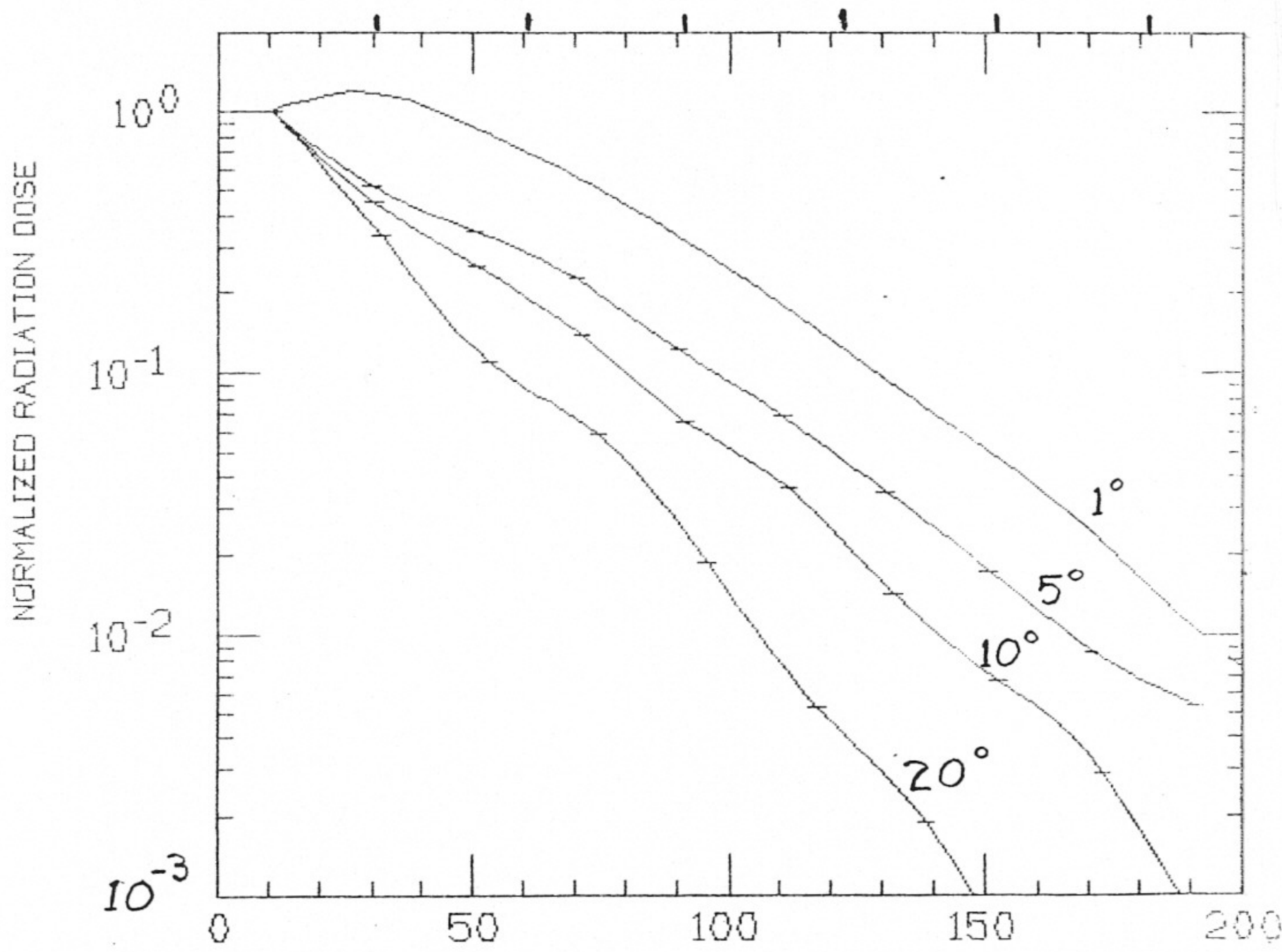

DEPTH IN CENTIMETERS

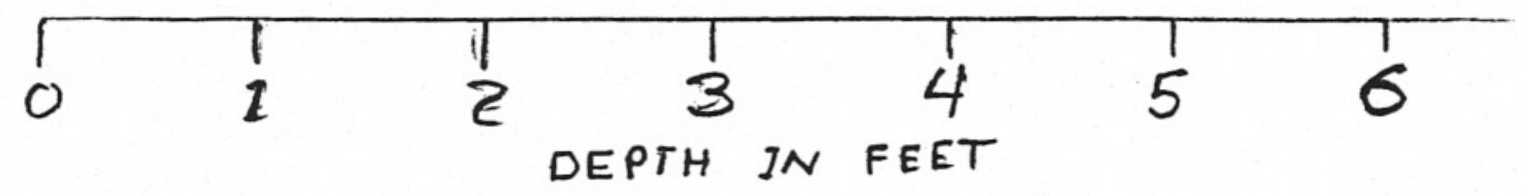

Fig. 4. Normalized radiation dose versus depth in steel for various angles of production. 